REPORT

\title{
Toll-like receptors in rheumatoid arthritis joint destruction mediated by two distinct pathways
}

\author{
C Ospelt, D Kyburz, M Pierer, R Seibl, M Kurowska, O Distler, M Neidhart, U Muller-Ladner, T Pap, \\ R E Gay, S Gay
}

$\mathrm{N}$ ovel therapies, in particular biological agents, have resulted in major breakthroughs in the treatment of rheumatoid arthritis (RA). However, none of the new and promising biologicals has resulted in an American College of Rheumatology (ACR) 70 above $50 \%$, and none of them has shown sustained benefit after termination of therapy. These results are based on the fact that all these agents target the inflammatory cells in the joint, including macrophages, T and B lymphocytes, or vascular endothelium but not the activated synovial fibroblasts. RA synovial fibroblasts (RA-SFs) have to be considered key cells in joint destruction since they differ in their morphology and their biological behaviour from normal synoviocytes and, most importantly, show invasive growth into adjacent tissue.

We have shown that RA-SFs maintain their activated phenotype independently of inflammatory cells and cytokines, considering that they invade human cartilage in the severe combined immunodeficiency (SCID) mouse model even in the absence of cytokine producing macrophages and $\mathrm{T}$ and $\mathrm{B}$ cells. ${ }^{1}$ By analysing cytokines, matrix degrading enzymes, and signalling molecules in the SCID mouse model, we explored the factors leading to synovial activation.

Characterisation of RA-SFs in detail has shown that cellular activation is driven and maintained by proinflammatory cytokines as well as by cytokine independent pathways of activation such as endogenous retroviral elements. In these studies, it was shown for the first time that a specific isoform of the p38 family, namely p38 is induced through the expression of Ll retrotransposable elements in RA-SFs in a cytokine independent pathway ${ }^{2} 3$ (fig 1). In RA synovial tissue, $\mathrm{p} 38 \delta$ is predominantly expressed at sites of invasion and bone destruction, whereas other members of the p38 family, such as p $38 \alpha$, are found in the non-attached lining and sublining layers.

These data and the results from the SCID mouse model clearly indicate that at least two pathways of joint destruction are operational in RA. ${ }^{4}$ The cytokine dependent pathway is mainly driven by inflammatory cells and targeted by the biologicals, whereas RA-SFs play a key role in the cytokine independent pathway.

Most recently, our work has focused on the search for additional stimulating factors in the activation of RA-SFs in RA. On the basis of the fact that bacterial DNA containing CpG motifs and bacterial cell wall fragments (peptidoglycans) have been detected in the synovial fluid of patients with RA, we have been searching for the presence of the germ line encoded pattern recognition receptors, called toll-like receptors (TLRs), to initiate activating signals in these cells. ${ }^{56}$

The TLR family comprises at least 11 members, however, TLR-11 was identified only very recently. ${ }^{7}$ Even though the diverse ligands and signalling pathways of most TLRs are well characterised, it is still not clear how and where exactly they interact with their ligands and whether they possibly contribute to the development of autoimmune diseases.

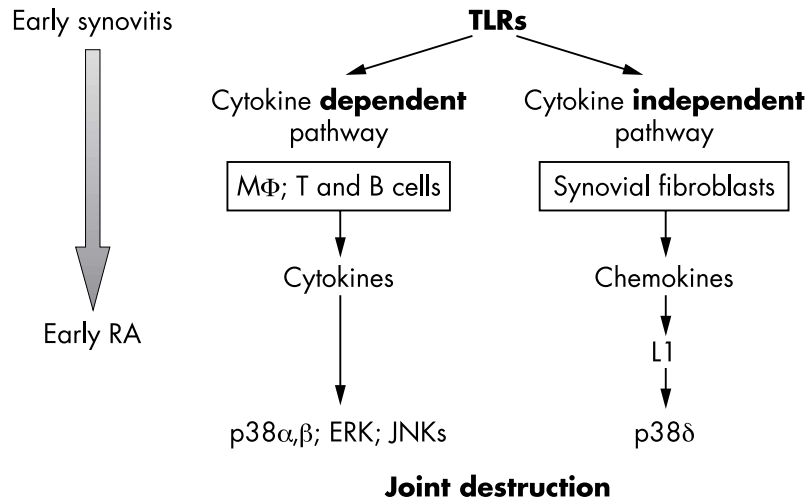

Figure 1 Cellular activation pathways in rheumatoid arthritis joint destruction. EKR, extracellular regulating kinase; JNK, c-Jun-N-terminal kinase; TLR, toll-like receptor.

We have shown that RA-SFs express TLR2 in culture and in the lining layer of synovial tissue, especially at sites of invasion into cartilage and bone. ${ }^{8}$ TLR 2 recognises a variety of microbial components, but it is critical for the recognition of peptidoglycans. In agreement with these data, cultured RASFs have been stimulated with peptidoglycans, but not with CpG DNA, which is a ligand for TLR9.' TLR2 expression in RA-SFs was upregulated by stimulation with proinflammatory cytokines and after exposure with synthetic lipopeptides and lipopolysaccharides.

More recently, we used microarray techniques to characterise genes that were induced in RA-SFs after stimulation with peptidoglycans. We demonstrated that TLR2 signalling in these cells results not only in the upregulation of nuclear factor $(\mathrm{NF})-\kappa \mathrm{B}$, certain matrix metalloproteinases, and cyclooxygenase-2, but also, most impressively, in the massive upregulation of several potent chemokines. ${ }^{10}$ These chemokines were subsequently detected in synovial tissue as well as in synovial fluid of patients with RA. Most striking is the fact that monocyte chemoattractant protein (MCP)-2 and granulocyte chemotactic protein (GCP)-2, which were among the most strongly induced genes, have never been investigated in arthritis before. Since both are found in significantly higher levels in the synovial fluid of patients with RA compared with patients with osteoarthritis, it can be presumed that these chemokines have an important role in the pathogenesis of RA.

A novel TLR2 fusion protein has been designed to block TLR2 mediated signalling and thus inhibit the activation of RA-SFs. Furthermore, new potential binding ligands can be identified by these means. ${ }^{6}$

Abbreviations: RA, rheumatoid arthritis; RA-SF, RA synovial fibroblast; $S C I D$, severe combined immunodeficiency, TLR, toll-like receptor 
Altogether, these data point to a cytokine independent pathway of RA-SF activation by TLR ligands that probably leads to chemoattraction of inflammatory cells at an early stage of disease. Through the expression of various TLRs, RA-SFs act as part of the innate immune system. At an early stage of the disease, they recognise microbial structures or yet unknown endogenous ligands and initiate an inflammatory response.

Of special interest is the observation that the expression of TLR2 and the downstream signalling molecules mediating joint destruction are mainly found at sites of synovial invasion into cartilage and bone, where oxygen supply is low. By examining the hypoxia induced cellular pathways, Kurowska and Distler demonstrated that hypoxia can be detected at sites of destruction in joints in RA. ${ }^{11}$ Furthermore, they showed that hypoxia induces a number of interesting molecules, including Id-2, a negative regulator of the basicHelix-Loop-Helix (bHLH) transcription factor. Id-2 is induced in RA-SFs by hypoxia and proinflammatory cytokines in a hypoxia inducible factor (HIF-1) independent way and mediates the upregulation of the receptor activator of NF- $\kappa B$ ligand (RANKL). Since RANKL promotes the differentiation of osteoclasts, this pathway potentially facilitates osteoclast mediated bone destruction in RA. ${ }^{12}$

\section{Authors' affiliations}

C Ospelt, D Kyburz, M Pierer, R Seibl, M Kurowska, O Distler, M Neidhart, U Muller-Ladner, T Pap, R E Gay, S Gay, WHO Collaborating Center for Molecular Biology and Novel Therapeutic Strategies for Rheumatic Diseases, Clinic for Rheumatology, University Hospital, Zurich, Switzerland

Correspondence to: S Gay, WHO Collaborating Center for Molecular Biology and Novel Therapeutic Strategies for Rheumatic Diseases, Clinic for Rheumatology, University Hospital, Gloriastrasse 25, CH-8091 Zurich, Switzerland; Steffen.Gay@usz.ch

\section{REFERENCES}

1 Muller-Ladner U, Kriegsmann J, Franklin BN, Matsumoto S, Geiler T, Gay RE, et al. Synovial fibroblasts of patients with rheumatoid arthritis attach to and invade normal human cartilage when engrafted into SCID mice. Am J Pathol 1996; 149:1607-15.

2 Kuchen S, Seemayer C, Rethage J, von Knoch R, Kuenzler P, Michel B, et al. The $\mathrm{L} 1$ retroelement-related p40 protein induces p38d MAP kinase. Autoimmunity 2004;37:57-65.

3 Neidhart M, Rethage J, Kuchen S, Kunzler P, Crowl RM, Billingham ME, et al. Retrotransposable L1 elements expressed in rheumatoid arthritis synovial tissue: association with genomic DNA hypomethylation and influence on gene expression. Arthritis Rheum 2000:43:2634-47.

4 Gay S, Gay RE, Koopman WJ. Molecular and cellular mechanisms of joint destruction in rheumatoid arthritis: two cellular mechanisms explain joint destruction? Ann Rheum Dis 1993;52(suppl 1):S39-S47.

5 van der Heijden IM, Wilbrink B, Tchetverikov I, Schrijver IA, Schouls LM, Hazenberg MP, et al. Presence of bacterial DNA and bacterial peptidoglycans in joints of patients with rheumatoid arthritis and other arthritides. Arthritis Rheum 2000;43:593-8.

6 Seibl R. Toll-like receptors: studies on cellular activation and involvement in rheumatoid arthritis. Zurich: ETH, 2003.

7 Zhang D, Zhang G, Hayden MS, Greenblatt MB, Bussey C, Flavell RA, et al. A toll-like receptor that prevents infection by uropathogenic bacteria. Science 2004;303:1522-6.

8 Seibl R, Birchler T, Loeliger S, Hossle JP, Gay RE, Saurenmann T, et al Expression and regulation of Toll-like receptor 2 in rheumatoid arthritis synovium. Am J Pathol 2003;162:1221-7.

9 Kyburz D, Rethage J, Seibl R, Lavener R, Gay RE, Carson DA, et al. Bacterial peptidoglycans but not $\mathrm{CpG}$ oligodeoxynucleotides activate synovial fibroblasts by toll-like receptor signaling. Arthritis Rheum 2003:48:642-50

10 Pierer M, Rethage J, Seibl R, Lavener R, Brentano F, Wagner U, et al. Chemokine secretion of rheumatoid arthritis synovial fibroblasts stimulated by Toll-like receptor 2 ligands. J Immunol 2004;172:1256-65.

11 Distler JH, Wenger RH, Gassmann M, Kurowska M, Hirth A, Gay S, et al. Physiologic responses to hypoxia and implications for hypoxia-inducible factors in the pathogenesis of rheumatoid arthritis. Arthritis Rheum 2004:50:10-23.

12 Kurowska M, Distler JH, Moritz W, Marti H, Gay R, Maslinski W, et al. The expression of inhibitor of differentiation-2 (Id-2) is induced by hypoxia in synovial fibroblasts independently of HIF-1a. Arthritis Rheum 2003;48(suppl 9):S146. 